\title{
BMJ Open Continuing education for the prevention of mild cognitive impairment and Alzheimer's-type dementia: a systematic review and overview of systematic reviews
}

\author{
Nina Matyas, ${ }^{\oplus 1}$ Filiz Keser Aschenberger, ${ }^{2}$ Gernot Wagner, ${ }^{1}$ Birgit Teufer, ${ }^{1}$ \\ Stefanie Auer, ${ }^{3}$ Christoph Gisinger, ${ }^{4}$ Monika Kil, ${ }^{2}$ Irma Klerings, ${ }^{1}$ \\ Gerald Gartlehner ${ }^{1,5}$
}

To cite: Matyas N, Keser Aschenberger F, Wagner G, et al. Continuing education for the prevention of mild cognitive impairment and Alzheimer'stype dementia: a systematic review and overview of systematic reviews. BMJ Open 2019;9:e027719. doi:10.1136/ bmjopen-2018-027719

- Prepublication history and additional material for this paper are available online. To view please visit the journal online (http://dx.doi.org/10.1136/ bmjopen-2018-027719).

Received 8 November 2018

Revised 24 May 2019

Accepted 6 June 2019

\section{Check for updates}

(C) Author(s) (or their employer(s)) 2019. Re-use permitted under CC BY-NC. No commercial re-use. See rights and permissions. Published by BMJ.

For numbered affiliations see end of article.

\section{Correspondence to} Dr. Nina Matyas; nina.matyas@ext.donau-uni. ac.at

\section{ABSTRACT}

Objective To summarise evidence on the preventive effects of continuing education on mild cognitive impairment and Alzheimer's-type dementia in adults 45 years or older.

Design Systematic review and overview of systematic reviews.

Data sources We systematically searched MEDLINE, PsycINF0, EMBASE, Cochrane Central Register of Controlled Trials, Cumulative Index to Nursing and Allied Health Literature, and Scopus for published studies and grey literature databases for unpublished studies from January 1990 to April 2018.

Methods To assess evidence directly addressing our objectives, we conducted a systematic review. Because we were aware of a dearth of direct evidence, we also performed an overview of systematic reviews on leisure activities that mimic formal continuing education. We a priori established the inclusion and exclusion criteria. Two authors independently assessed inclusion and exclusion at the abstract and full-text level, rated the risk of bias, and determined the certainty of evidence using the Grading of Recommendations Assessment, Development and Evaluation. We resolved all discrepancies by consensus. We synthesised the available evidence narratively. Results Our searches identified 4933 citations. For the systematic review, only two publications on the same prospective cohort study (Tasmanian Healthy Brain Project) met the inclusion criteria; for the overview of reviews, we included five systematic reviews. Based on 459 participants, preliminary data of the ongoing cohort study indicated that cognitive reserve statistically significantly increased in persons attending university classes compared with the control group $(92.5 \%$ vs $55.7 \%, p<0.01)$. Likewise, language processing capacities statistically significantly improved $(p<0.01)$. Episodic memory, working memory and executive function did not differ significantly between groups. Systematic reviews consistently reported a positive association between participation in cognitively stimulating leisure activities and reduced incidence of dementia and improved cognitive test performance.
Strengths and limitations of this study

- This is the first systematic review assessing the benefits and harms of continuing education on the prevention of mild cognitive impairment or Alzheimer's-type dementia

- This is the first overview of systematic reviews presenting an up-to-date summary of currently available research in the field of cognitive leisure activities and dementia.

- The certainty of evidence is low, indicating that future studies might have a substantial impact on the results of our review.

- Measurements and types of cognitive leisure activities differed widely across studies and quantitative analyses were often not possible.

- The majority of included systematic reviews have serious methodological shortcomings.

Conclusion Available results demonstrate that cognitive reserve increases through continuing education and show a positive association of cognitive leisure activities with both improved cognitive function and lower dementia incidence.

PROSPERO registration number CRD42017063944.

\section{INTRODUCTION}

In 2012, the WHO named the prevention and control of neurocognitive disorders such as mild cognitive impairment (MCI) or Alzheimer's-type dementia a global public health priority. ${ }^{1}$ Alzheimer's-type dementia is the most common form of dementia. ${ }^{1}$ In 2018, 50 million people worldwide lived with Alzheimer's disease or another closely related form of dementia. ${ }^{2}$ As a consequence of the rapidly ageing world population, the prevalence of dementia is projected to rise up to 152 million people in $2050 .^{2}$ 
The progressive loss of independent functioning of people with Alzheimer's-type dementia leads to an enormous social and economic burden. In 2018, the US economic burden associated with Alzheimer's-type dementia was estimated to be US $\$ 277$ billion. ${ }^{3}$ The total global costs for dementia were about a trillion US dollars in $2018 .^{2}$

The Diagnostic and Statistical Manual-5 characterises Alzheimer's disease as a significant decline of intellectual abilities in one or more cognitive domains (learning and memory, language, executive function, complex attention, perceptual motor function, and social cognition) outside the context of delirium. ${ }^{4}$

Any dementia diagnosis, however, is frequently preceded by a long period of subclinical neuropathological disorder with subjective cognitive disorder and mild cognitive disorder as a transition phase before diagnostic criteria for dementia are fulfilled. ${ }^{5}$ If cognitive decline progresses to a degree that a person's capability of carrying out everyday activities is significantly affected, this state is called major neurocognitive disorder. ${ }^{45}$

The risk of developing neurocognitive disorders increases substantially with age. The prevalence of Alzheimer's-type dementia is $3.5 \%$ in persons aged 75 or older and $46.3 \%$ in those 95 years or older. ${ }^{6}$ It is estimated that $15 \%-20 \%$ of people age 65 or older are living with MCI. ${ }^{3}$ Other risk factors than age contributing to the development of dementia are not yet thoroughly understood. In recent years, epidemiological studies have linked the development of dementia with risk factors such as low educational level, unhealthy diet, decreased physical activity and smoking. ${ }^{78}$ In addition, potential predictors of dementia are chronic medical conditions such as cardiovascular diseases, diabetes, obesity, cancers, depression, thyroid disorder or genetic factors. ${ }^{9}$ Some studies, however, found a protective association of cognitively stimulating activities, such as learning a new language in middle age, with a slower cognitive decline during late life ${ }^{10-14}$ Such results underpin the "cognitive reserve hypothesis'. ${ }^{15-17}$ According to this theory, through every activity that stimulates the brain, the cognitive reserve gets boosted and the resistance towards any dementia-related brain pathology increases. ${ }^{18}$ In animal trials, an enriched environment was associated with increased cortical thickness. ${ }^{19}$ Epidemiological research on humans has shown that education ${ }^{20}$ and probably also other forms of intellectual stimulation, during the whole lifespan, are associated with a lower risk to develop dementia. ${ }^{21}{ }^{22} \mathrm{~A}$ larger cognitive reserve acquired by continuing education activities, thus, might protect against cognitive decline. ${ }^{1823}$

Continuing education activities are structured learning activities offered by educational institutes. These activities are designed to help individuals satisfy learning needs and interests after compulsory schooling, to enrich knowledge, to develop and improve abilities and skills, and to foster personality, social competences, families, networks, health and professional life. Continuing education is voluntary, based on topics and courses that are not directly connected to any special job position or vocational training. ${ }^{24-29}$
Cognitive leisure activities (eg, learning a new language) often mimic continuing education activities but are not taking place within the framework of an educational institution.

\section{Rationale}

To date, the preventive effect of continuing education on cognitive impairment and Alzheimer's-type dementia has not been assessed in an objective and systematic way. The aim of our review was to summarise the evidence investigating the preventive effects of continuing education on the development of cognitive impairment and Alzheimer's-type dementia.

Our systematic review addressed the following questions:

- Key question 1a: In adults 45 years of age or older with normal cognition or merely subjective cognitive impairment, does continuing education lead to a reduction in the risk of MCI or Alzheimer's-type dementia compared with no continuing education?

- Key question $1 b$ (in case no evidence on continuing education is available or the evidence is sparse): In adults 45 years of age or older with normal cognition or merely subjective cognitive impairment, do cognitive leisure activities lead to a reduction in the risk of MCI or Alzheimer's-type dementia compared with no cognitive leisure activities?

- Key question 2: What are the potential harms of continuing education?

- Key question 3: Do benefits and harms differ by subgroups based on age, sex/gender, race or ethnicities, level of education, or duration of intervention?

- Key question 4: What is the optimal age to start continuing education to prevent MCI or dementia?

\section{METHODS}

\section{Design}

Throughout this manuscript we followed the Preferred Reporting Items for Systematic Reviews and Meta-Analyses statement ${ }^{30}$ (see online supplementary file 1 ). The protocol of this systematic review was registered in PROSPERO (International Prospective Register of Systematic Reviews) and published previously. ${ }^{31}$ Figure 1 depicts the analytic framework that guided our systematic review.

For this systematic review, we define continuing education as structured learning activities and programmes provided by formal and non-formal educational institutions for persons beyond the age of compulsory schooling (in most countries 16 years and older). ${ }^{24-29}$

We addressed our research questions with two different methodological approaches:

1. We performed a systematic review of primary studies to assess the preventive effects and potential harms of continuing education provided by formal and non-formal institutions (key questions 1a, 2, 3 and 4).

2. We conducted an overview of systematic reviews to determine the preventive effects and potential harms 


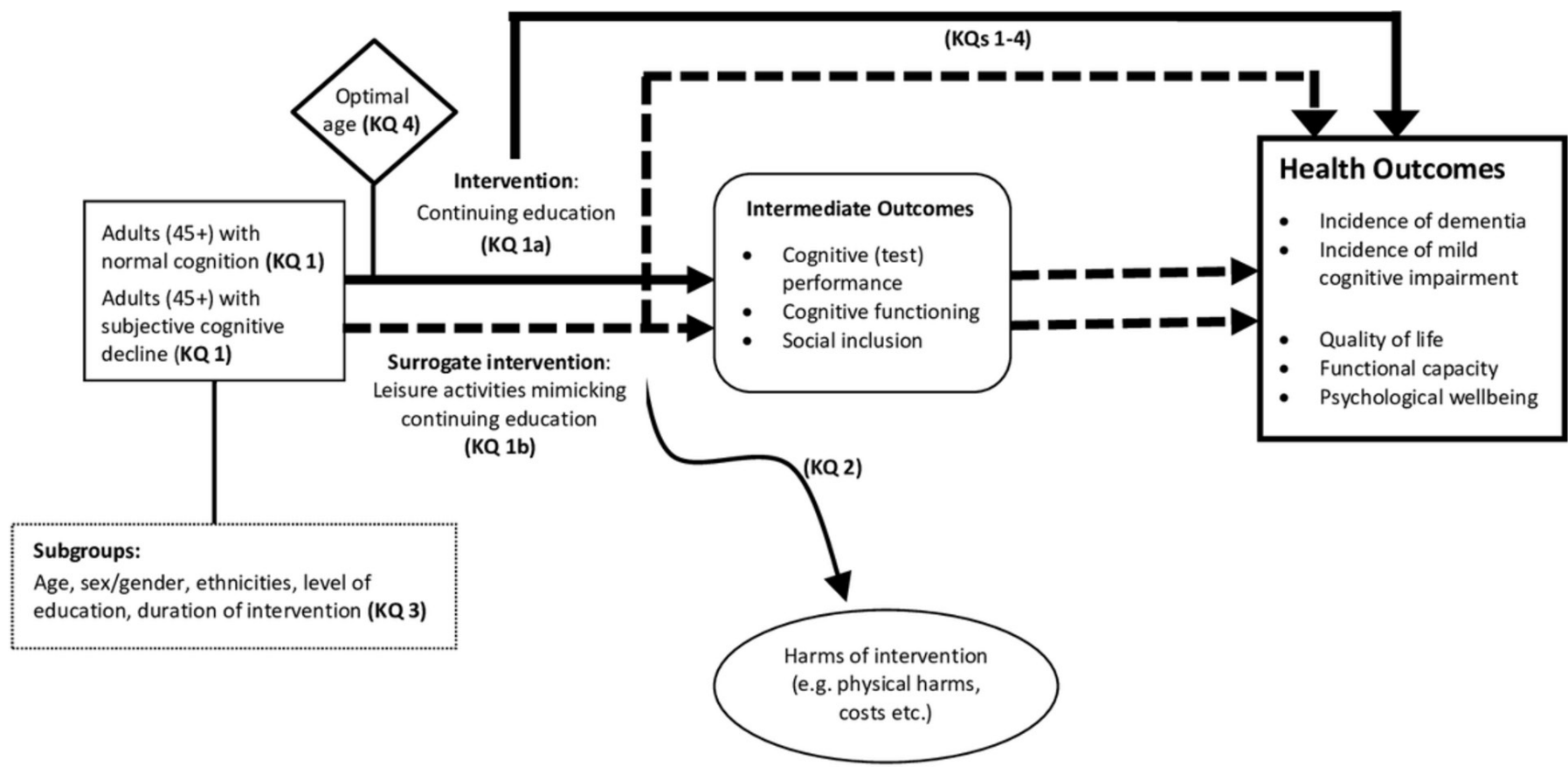

Figure 1 Analytic framework for continuing education to prevent mild cognitive impairment and Alzheimer's-type dementia. $K Q$, key question.

of related leisure activities (eg, playing cards, reading

books and so on; key question 1b).

We chose this two-step approach because studies in the field of continuing education and dementia are very rare. Certain leisure activities, however, are able to mimic continuing education regarding content (eg, learning a new language privately vs learning a new language as an organised educational activity). Leisure activities are not our primary focus of interest but can be considered as proxy interventions for continuing education in some circumstances.

\section{Study selection}

Eligibility criteria for the systematic review

The population of interest were adults 45 years or older without a clinical diagnosis of cognitive impairment at the time of study recruitment, which included people with subjective cognitive impairment. Eligible interventions comprised all cognitive activities that are provided by formal and non-formal educational institutions. These activities include classes, courses and trainings that are based on individual interests and that are attended voluntarily. We included randomised controlled trials, non-randomised controlled trials, prospective controlled cohort studies, retrospective controlled cohort studies and casecontrol studies. All non-randomised studies needed to have a minimum sample size of 300 or more participants.

Eligible studies had a minimum follow-up time of 1 year and a minimum duration of intervention of 3 months. We excluded studies that investigated formal (vocational) education (eg, school or college), physical activities, and all job-related courses and trainings.

Outcomes of interest included patient-relevant health outcomes such as incidence of dementia, incidence of
MCI, psychological well-being, functional capacity, quality of life and other relevant health outcomes; in addition, we included intermediate outcomes such as cognitive functioning, cognitive (test) performance or social inclusion. For the purpose of our study, mild cognitive impairment refers to 'amnestic' mild cognitive impairment, meaning that memory loss is the predominant symptom. ${ }^{32}$

\section{Eligibility criteria for the overview of systematic reviews}

Eligibility criteria for population and outcomes for the overview of systematic reviews were the same as for the systematic review. Eligible interventions were leisure activities that are cognitively stimulating and mimic the content of continuing education but in an informal setting. Just as in the systematic review, we excluded physical activities. Eligible study designs were exclusively systematic reviews and meta-analyses. We excluded reviews with searches conducted before 2013 .

Further details about our inclusion and exclusion criteria can be found in our protocol ${ }^{31}$ and in online supplementary file 2 .

\section{Search strategy}

We systematically searched Ovid MEDLINE, Cochrane Library, EMBASE, PsycINFO, Cumulative Index to Nursing and Allied Health Literature, ALOIS (the Cochrane Dementia and Cognitive Improvement Group Specialised Register) and Education Resources Information Center from January 1990 to April 2018 to identify relevant publications (see online supplementary file 3 for the search strategy). For the overview of reviews, we searched Epistemonikos from inception to April 2018 in addition to the above-mentioned databases (see online supplementary file 4 ). 
An experienced information specialist developed an appropriate search strategy using a combination of medical subject headings and title and abstract keywords, limiting the search to human-only studies without applying any language limitations. The electronic Ovid MEDLINE search strategy was peer-reviewed by another information specialist following the PRESS (Peer Review of Electronic Search Strategies) statement. ${ }^{33}$ For the systematic review, we searched for grey literature in ClinicalTrials.gov, WHO's International Clinical Trials Registry Platform, web pages of relevant organisations and a dissertation database ('Digital Access to Research Theses', DART-Europe). Additionally, in an attempt to avoid retrieval bias, we manually searched the reference lists of landmark studies and background articles on this topic to look for any relevant citations that our electronic searches might have missed. We imported all citations into an electronic database (EndNote V.X8) and deleted duplicates.

\section{Study selection}

Two review authors independently screened the abstracts and relevant full-text articles for eligibility, using Covidence software. ${ }^{34}$ They resolved disagreements by discussion or by consultation with a third author.

\section{Data abstraction}

We designed, pilot-tested and used a data abstraction form to gather pertinent information from each article. One author extracted relevant data from each study that met our inclusion criteria. A second author of the team cross-checked data abstractions for completeness and accuracy. We extracted study information (author, publication year, years covered by searches, location/ setting, number of included studies and included study designs), sample size, study characteristics (population, interventions and comparators), outcome measurements and results. For systematic reviews we abstracted summary estimates of meta-analyses whenever available.

\section{Risk of bias assessment}

Two investigators independently assessed the risk of bias of included studies. They resolved any disagreements by consensus or by consulting a third team member. For eligible non-randomised studies, we used the Risk Of Bias In Non-randomised Studies of Interventions tool. ${ }^{35}$ For the assessment of eligible systematic reviews, we used the Assessing the Methodological Quality of Systematic Reviews tool. ${ }^{36}$ Detailed risk of bias ratings of included articles are given in online supplementary file 5 .

\section{Data synthesis and statistical analysis}

We narratively summarised evidence from included studies. If available, we present effect estimates of systematic reviews. For the incidence of dementia, we present HR, OR and risk ratio (RR). For the assessment of cognitive test performance, we use standardised mean differences (SMDs) because scales for measurements differed in the individual studies. An SMD of 0 indicates that both groups had the same cognitive test performance.

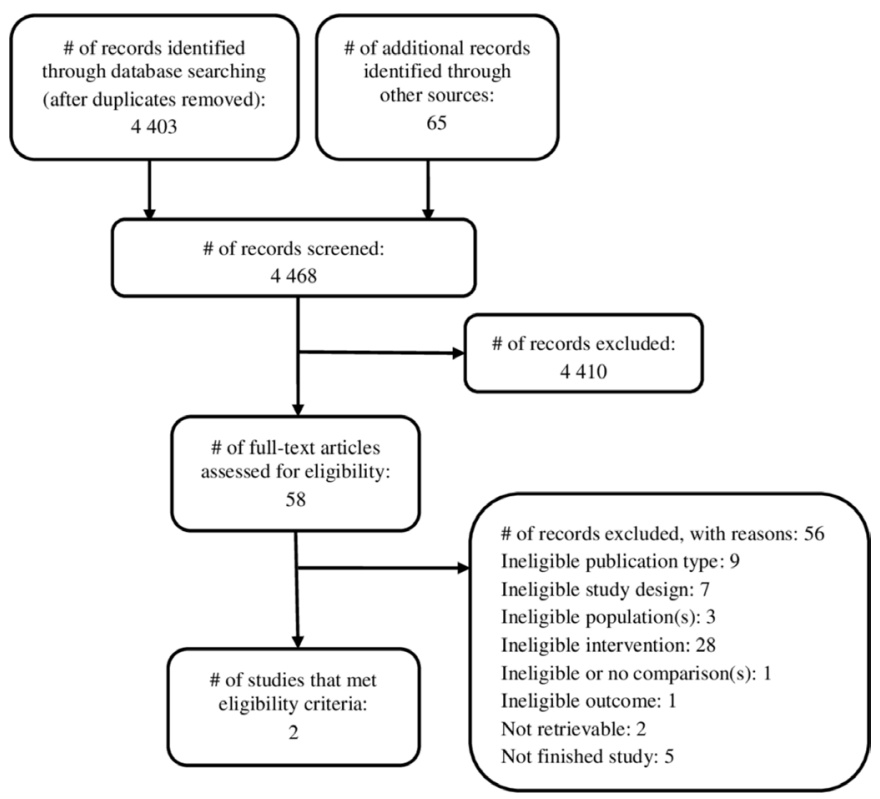

Figure 2 Flow diagram of systematic review of continuing education for the prevention of mild cognitive impairment and Alzheimer's-type dementia.

\section{Certainty of evidence}

We followed the recommendations of the Grading of Recommendations Assessment, Development and Evaluation working group for rating the certainty of evidence for each outcome. ${ }^{37}$

\section{Patient involvement}

No patients were involved in the development of this research paper.

\section{RESULTS}

Our search identified in total 4933 citations after exclusion of duplicates. Based on title and abstract review, we considered 58 primary studies and 28 systematic reviews for full-text review. After scrutinising the full-text articles, we included two publications of one primary study ${ }^{38} 39$ and five systematic reviews. ${ }^{40-44}$ Figure 2 and figure 3 depict the study selection process. Online supplementary file 6 provides a list of excluded studies at the full-text level.

\section{Study characteristics}

\section{Systematic review}

We included two publications ${ }^{38} 39$ that present interim findings of the same medium risk of bias prospective cohort study, namely the Tasmanian Healthy Brain Project, which plans to follow participants for 10-20 years. ${ }^{45}$ The two publications analysed different aspects of cognitive functioning of the same 459 participants who did or did not engage in a 12-month, part-time or fulltime, university-level education. Participants' mean age was $59.6 \pm 7($ mean $\pm \mathrm{SD})$ years in the intervention group and $62.4 \pm 6$ years in the control group, with a follow-up period of 4 years. Participants completed a neuropsychological test battery, consisting of 14 tests each year. ${ }^{45}$ 


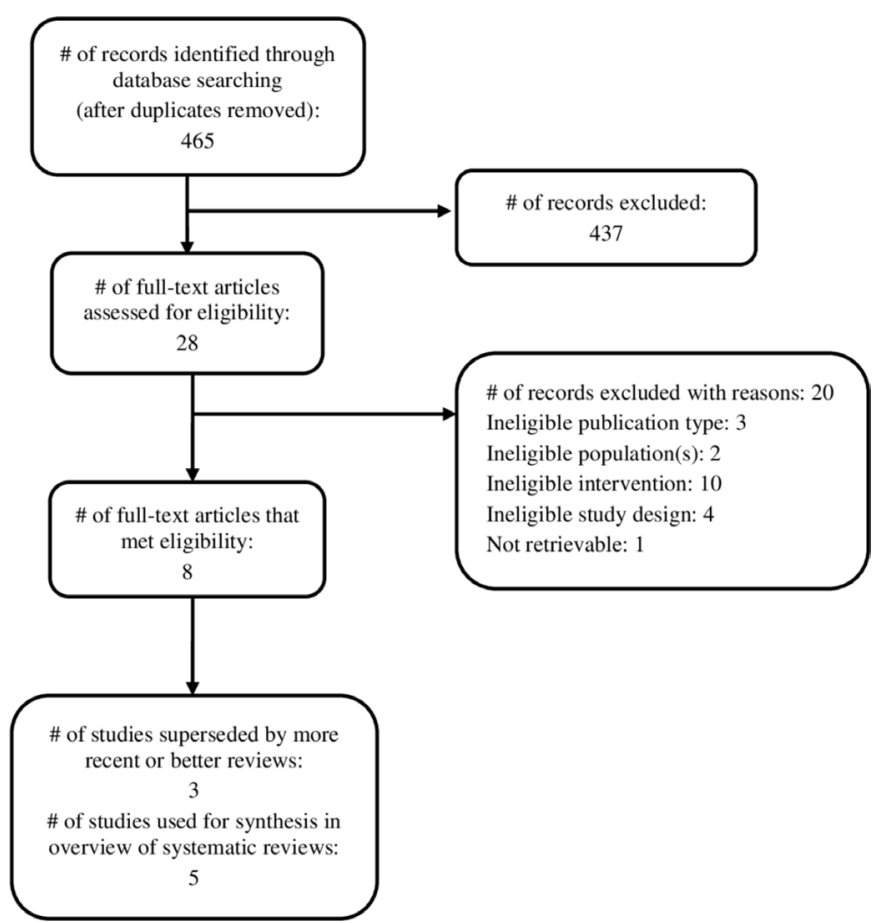

Figure 3 Flow diagram of overview of systematic reviews of cognitive leisure activities for the prevention of mild cognitive impairment and Alzheimer's-type dementia.

In this study, selection bias is likely because participants voluntarily opted for university courses or no further education. It is not clear if investigators used appropriate methods to adjust for potential confounders (see online supplementary file 5 for risk of bias ratings).

\section{Overview of reviews}

All five included systematic reviews investigated the benefits of cognitive leisure activities in adults over the age of 45 years. ${ }^{40-44}$ Four studies included any cognitive leisure activities in their analyses (eg, reading books, doing crosswords, attending cultural events, knitting, painting), and one study ${ }^{43}$ specifically focused on the benefits of playing video games in older adults. The number of participants investigated in the reviews ranged from $913^{43}$ to $24554 .^{41}$

We rated three of the included systematic reviews ${ }^{4042} 43$ as high and two studies ${ }^{414}$ as medium risk of bias. Reasons for the high risk of bias ratings were lack of critical appraisal of included studies, single review of the literature and insufficient literature searches (see online supplementary file 5).

We graded the evidence for all meta-analysis outcomes with low certainty of evidence mostly because of high inconsistency and indirectness among studies (see online supplementary file 7 ).

We present the characteristics of included studies ${ }^{40-44}$ in table 1 and table 2.

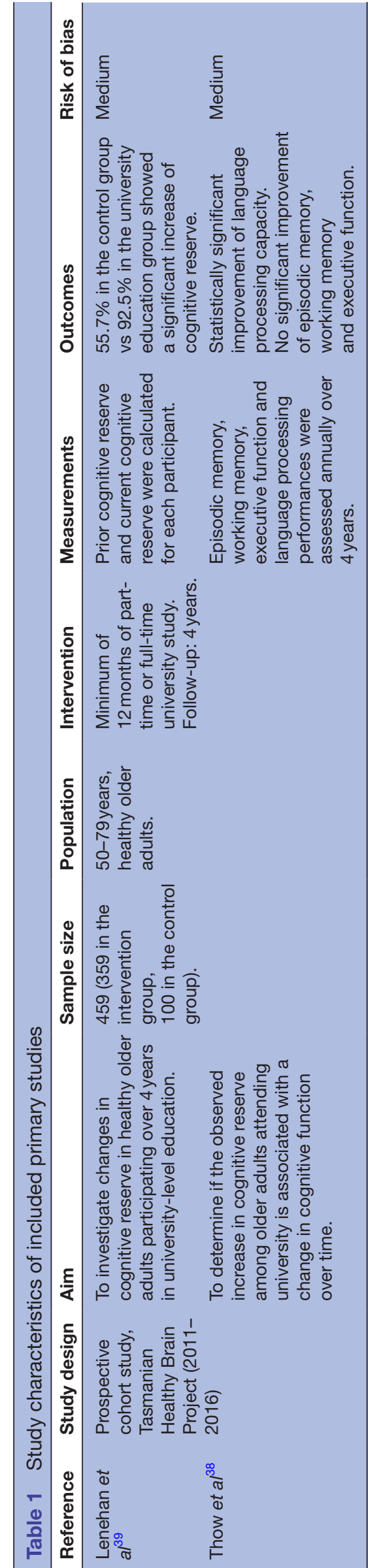




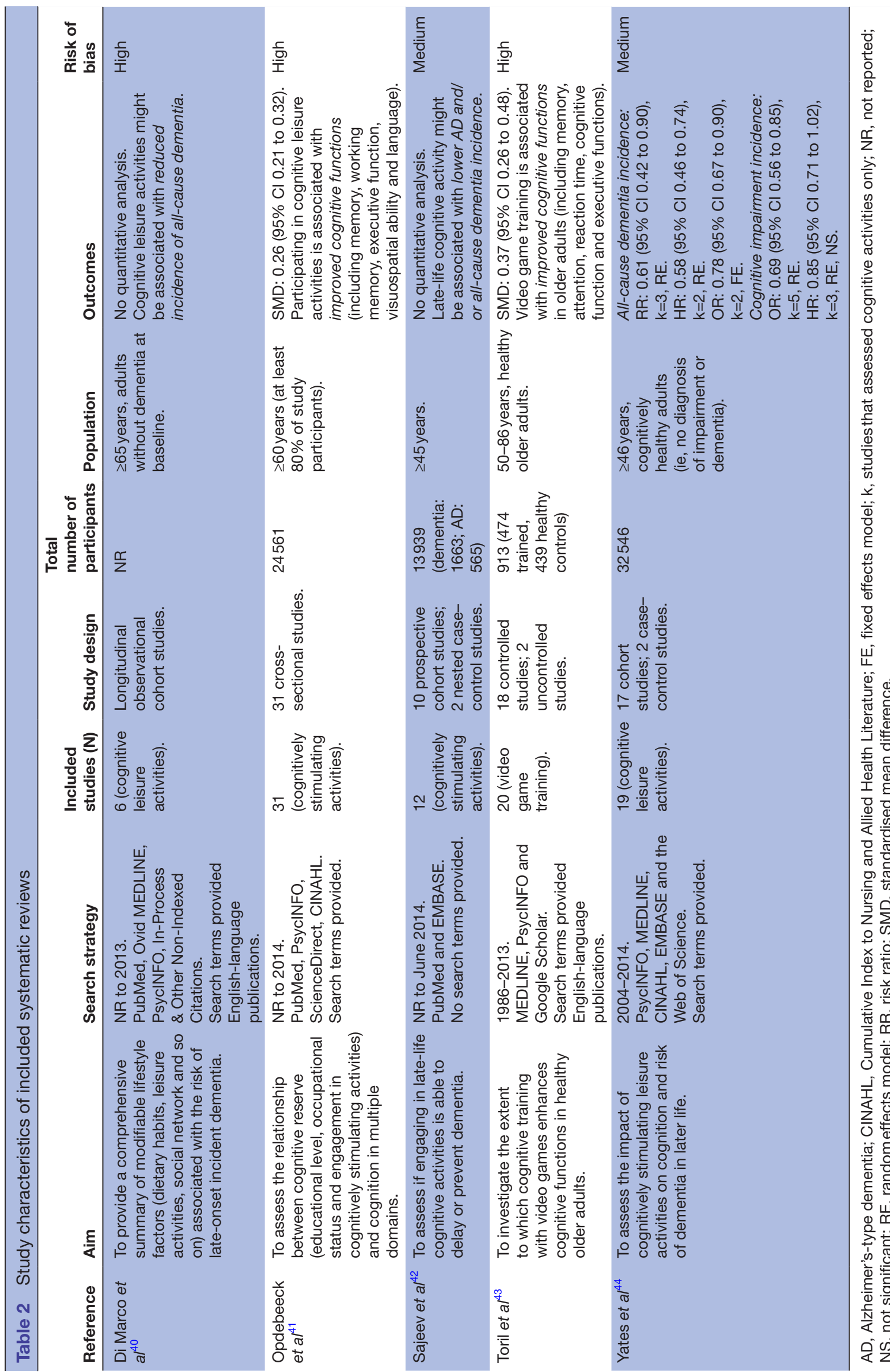




\section{Outcomes}

Key question 1a: continuing education

Two interim analyses of the Tasmanian Healthy Brain Project focused on language processing ${ }^{38}$ and cognitive reserve $^{39}$ after 4 years of follow-up. To date, no results on the incidence of MCI or Alzheimer's-type dementia are available yet. Both studies reported beneficial effects of continuing education. Thow and colleagues ${ }^{38}$ showed that attending university courses over a period of 12 months statistically significantly $(\mathrm{p}<0.05)$ improved the language processing capacity in the intervention group compared with the control group. No statistically significant differences were detected for episodic memory, working memory and executive function between groups. In all analyses, authors accounted for age and prior cognitive reserve (education, pre-existing intellectual capacity, life experience).

Lenehan and coworkers ${ }^{39}$ demonstrated by conducting growth mixture modelling that the cognitive reserve statistically significantly increased in $92.5 \%$ of participants in the intervention group $(n=359)$ compared with $55.7 \%$ of participants in the control group $(n=100)$. Investigators created a proxy measure of 'current cognitive reserve' to capture dynamic changes in cognitive reserve over time, including intellectual capacity and academic ability. ${ }^{46}$

\section{Key question 1b: cognitive leisure activities}

Overall, the five included systematic reviews reported consistently that participation in cognitively stimulating leisure activities can reduce the risk of developing MCI or Alzheimer's-type dementia and improves cognitive functioning of healthy older adults.

Two systematic reviews ${ }^{40}$ investigated the impact of cognitive leisure activities on the incidence of Alzheimer's-type dementia. Di Marco and colleagues ${ }^{40}$ included 6 and Sajeev and colleagues ${ }^{42} 12$ primary studies on cognitive leisure activities in their systematic reviews. Both systematic reviews concluded that leisure activities protect against dementia. Due to different categorisation of cognitive leisure activities and a high heterogeneity between studies, quantitative analyses were not possible in the two reviews. The effect estimates of included studies ranged from an HR of $0.39(95 \% \text { CI } 0.21 \text { to } 0.71)^{47}$ to an HR of $0.93(95 \%$ CI 0.88 to 0.98$),{ }^{48}$ showing statistically significantly reduced risks of Alzheimer's-type dementia when carrying out leisure activities. Sajeev et $a t^{42}$ performed an extensive bias analysis indicating that it is unlikely that the observed positive effects of cognitively stimulating activities on dementia incidence are exclusively explained by unmeasured confounders or reverse causation.

One review ${ }^{44}$ assessed both the incidence of MCI and Alzheimer's-type dementia and cognitive test performance. The authors conducted five meta-analyses based on groups for outcomes and reported effect estimates (RR, OR and HR). Four out of five meta-analyses revealed statistically significant results showing that cognitive leisure activities were associated with a reduction of dementia incidence $(\mathrm{RR}=0.61,95 \% \mathrm{CI} 0.42$ to 0.90 ;
$\mathrm{HR}=0.58,95 \%$ CI 0.46 to $0.74 ; \mathrm{OR}=0.78,95 \%$ CI 0.67 to $0.90)$ and a reduction of cognitive impairment incidence ( $\mathrm{OR}=0.69,95 \%$ CI 0.56 to 0.85$)$. However, one meta-analysis combining three cohort studies did not reach statistical significance $(\mathrm{HR}=0.85,95 \%$ CI 0.71 to 1.02$)$ for reduction of cognitive impairment. A narrative analysis of primary studies assessing cognitive test performance showed a statistically significant improvement of memory, speed of processing, language and executive functioning, and overall later life cognition.

Two other included studies, ${ }^{41} 43$ both rated as high risk of bias, focused on cognitive test performance. Opdebeeck et $a t^{41}$ reported a benefit in overall cognitive abilities for the group involving in cognitive leisure activities (SMD of $0.26,95 \%$ CI 0.21 to 0.32 ). The cognitive domains included memory, working memory, executive function, visuospatial ability and language. According to the review by Toril $e t a l,{ }^{43}$ playing video games enhanced several cognitive functions. They observed an SMD of 0.37 (95\% CI 0.26 to 0.48 ) for global cognitive function (combining results for memory, attention, reaction time and executive functions), showing a benefit for the intervention group.

\section{Key question 2: harms}

We found no evidence regarding harms of continuing education or cognitive leisure activities.

\section{Key question 3: subgroups}

Toril and colleagues ${ }^{43}$ performed several subgroup analyses. The study revealed that the age of the participants and the number of video game training sessions significantly changed the effect size. Older participants (between 71 and 80 years) seemed to benefit more from computer training than younger participants (60-70 years). For the improvement of cognitive test performance, shorter training sessions (1-6 weeks) seemed to show an advantage over longer training interventions (7-12 weeks). By contrast, for incidence of Alzheimer's-type dementia, Di Marco et $a t^{40}$ and Sajeev et $a t^{42}$ infer from their data that greater participation in cognitive leisure activities over a longer period of time contributes positively to the protective effect.

\section{Key question 4: optimal age}

No study specifically discussed the optimal age to start with continuing education activities or cognitive leisure activities to prevent MCI or dementia.

\section{DISCUSSION}

The evidence assessing the impact of continuing education on the risk of MCI or Alzheimer's-type dementia is limited. The only eligible primary study (Tasmanian Healthy Brain Project) ${ }^{45}$ is still ongoing, but preliminary findings after 4 years of follow-up demonstrated that the dynamic nature of cognitive reserve permits improvements through education even at an advanced 
age. Nevertheless, these early findings have to be viewed cautiously. In addition, selection bias could potentially distort the results of the Tasmanian Healthy Brain Project because participants voluntarily opted for university courses or no further education. The available publications did not explain sufficiently how baseline differences such as comorbid diseases were taken into consideration during the analyses.

Because of the limited direct evidence, we focused on cognitive leisure activities as proxies for formal continuing education. Overall, the available evidence consistently indicates beneficial effects of cognitive leisure activities by improving the cognitive function of older adults and reducing the incidence of MCI and Alzheimer's-type dementia. These findings could be explained by the neuroplasticity of the human brain, which refers to the ability of the brain to adapt to every new stimulus by forming dendritic connections, creating morphological changes and increasing cognitive reserve. ${ }^{49-51}$ Neuroplasticity is an intrinsic property of the human brain that allows us to learn and adapt to environmental changes. Depending on the stimuli, the changes can be positive or negative ${ }^{49-51}$ The concept of neuroplasticity is interrelated with the concept of cognitive reserve, which refers to morphological changes that support cognitive functioning. ${ }^{51}$

The magnitudes of beneficial effects, however, varied across systematic reviews, and CIs encompassed effect sizes that would not be clinically relevant. Consequently, we rated the certainty of evidence as low or very low, which means that future studies are likely to have a substantial impact on these findings.

To the best of our knowledge, our study was the first assessing the impact of continuing education on MCI and Alzheimer's-type dementia. It is also the first overview of systematic reviews presenting an up-to-date summary of currently available research in the field of cognitive leisure activities and dementia. Nevertheless, our work has several limitations. First, in the overview of systematic reviews, we had to rely on the quality of included systematic reviews which often had methodological shortcomings (eg, no risk of bias assessment, no dual screening and so on). Second, most of the included studies were observational studies, which are prone to selection bias because participants self-select the group. Risk factors for MCI or dementia in participants selecting leisure activities or further education might be systematically different from participants in the control groups. For example, people who eventually suffer from preclinical dementia stages might be more likely to avoid cognitive leisure activities, which would lead to reverse causation. Third, many primary studies within the reviews used self-reported questionnaires that could be challenging for people who start having cognitive deficits. Finally, the variation of leisure activity categorisation across studies made meta-analysis difficult and sometimes impossible. For example, 'visiting a library' was classified as a cognitive activity by one author but as a physical activity by another.
Some studies assessed current participation in activities, others participation at younger ages. Additionally, some studies assessed the frequency of participation, others the time devoted to activities and some the total number of leisure activities. Consequently, due to these limitations, the comparability of results among studies was limited. A standardisation of measures and methods would be necessary to help synthesise evidence in the future and make more reliable recommendations.

\section{Implication for future research, policy and practice}

Based on the preliminary results of a long-term cohort study and indirect evidence from studies on leisure activities, continuing education might be a promising option to help prevent dementia. A recent study suggests that modifiable risk factors (low education, midlife hypertension, midlife obesity, diabetes, physical inactivity, smoking and depression) might be responsible for about a third of Alzheimer's-type dementia cases.$^{52}$ Hence, considering our results, a campaign promoting to 'actively use the brain by participating in the wide range of continuing education' could possibly be added to the list of preventive options and could have an impact on the reduction of Alzheimer's-type dementia cases. The Finnish Geriatric Intervention Study to Prevent Cognitive Impairment and Disability, ${ }^{53}$ a randomised controlled trial with a multidomain approach (diet, exercise, cognitive training, vascular risk monitoring), supports the hypothesis that simultaneous changes in several risk factors can lead to a protective effect on cognition.

Further research is needed to address the evidence gap regarding continuing education and the extent to which it acts as a protective factor. A study similar to the Tasmanian Healthy Brain Project, ${ }^{39}$ but conducted as a randomised controlled trial, would be ideal because it would adequately handle known and unknown confounders. Computers and internet could play a more significant role in future prevention trials. Older adults could, for instance, be randomised to attend online courses and communicate with professors and other students in virtual classrooms. This approach could save time and money, and increase the potential participant pool.

\section{CONCLUSION}

Although no firm conclusions about the effects of continuing education on preventing MCI and dementia can be drawn, data from preliminary and indirect evidence indicate that continuing education could potentially have important preventive effects.

\section{Author affiliations}

${ }^{1}$ Department for Evidence-based Medicine and Clinical Epidemiology, DonauUniversitat Krems, Krems, Austria

${ }^{2}$ Department for Continuing Education Research and Educational Management, Donau-Universitat Krems, Krems, Austria

${ }^{3}$ Department for Clinical Neurosciences and Preventive Medicine, Donau-Universitat Krems, Krems, Austria 
${ }^{4}$ Center for Geriatric Medicine and Geriatric Nursing, Donau-Universitat Krems, Krems, Austria

${ }^{5}$ Evidence-based Practice Center, RTI International, Research Triangle Park, North Carolina, USA

Acknowledgements We would like to thank Manuela Müllner from Danube University Krems for administrative support.

Contributors All authors made substantial contribution to the conception and design of this study. GG, NM, MK, CG and SA developed the concept of the study. As an information specialist, IK developed the search strategy. GW, FKA, NM and BT conducted the literature review, abstracted the data and graded the strength of evidence. NM wrote the first draft of the manuscript; all authors reviewed the manuscript and provided comments. All authors have given approval for this version to be published.

Funding This work was supported by internal funds from Danube University Krems, Austria. It did not receive financial support from any funding agency in the public, commercial or not-for profit sectors.

Competing interests None declared.

Patient consent for publication Not required.

Provenance and peer review Not commissioned; externally peer reviewed.

Data sharing statement All extracted data are included in the manuscript. There are no additional data sets available.

Open access This is an open access article distributed in accordance with the Creative Commons Attribution Non Commercial (CC BY-NC 4.0) license, which permits others to distribute, remix, adapt, build upon this work non-commercially, and license their derivative works on different terms, provided the original work is properly cited, appropriate credit is given, any changes made indicated, and the use is non-commercial. See: http://creativecommons.org/licenses/by-nc/4.0/.

\section{REFERENCES}

1. World Health Organistation (WHO). Dementia: A public health priority. 2012 http://apps.who.int/iris/bitstream/10665/75263/1/ 9789241564458_eng.pdf?ua=1 (Accessed 20 Apr 2017).

2. Patterson C, Lynch C, Bliss A, et al. World Alzheimer report 2018 the state of the art of dementia research: New frontiers. $2018 \mathrm{https} / / /$ www.alz.co.uk/research/WorldAlzheimerReport2018.pdf (Accessed 15 Feb 2019).

3. Alzheimer's Association. Alzheimer's disease facts and figures. 2018 https://www.alz.org/media/HomeOffice/Facts and Figures/facts-andfigures.pdf (Accessed 15 Feb 2019).

4. Association AP. Diagnostic and statistical manual of mental disorders. Fifth Edition (DSM-5): American Psychiatric Publishing, 2013.

5. Committee on Decreasing the Risk of Alzheimer's-Type Dementia. Mild cognitive impairment age-related cognitive IBoHS, Policy Institute of, Medicine National Academies of Sciences, Engineering Medicine. The National Academies Collection: Reports funded by National Institutes of Health. Considerations for the Design of a Systemic Review of Interventions for Preventing Clinical Alzheimer'sType Dementia, Mild Cognitive Impairment, and Age-Related Cognitive Decline: Letter Report. 2015 https://www.nap.edu/catalog/ 21885/considerations-for-the-design-of-a-systematic-review-ofinterventions-for-preventing-clinical-alzheimers-type-dementia-mildcognitive-impairment-and-age-related-cognitive-decline (Accessed 17 Apr 2017).

6. Reynish E, Fratiglioni L, Prince M, et al. EUROCODE prevalence of dementia in Europe. 2006 https://ec.europa.eu/health/archive/ph information/dissemination/diseases/docs/eurocode.pdf (Accessed 6 Mar 2017)

7. Alonso A, Jacobs DR, Menotti A, et al. Cardiovascular risk factors and dementia mortality: 40 years of follow-up in the Seven Countries Study. J Neurol Sci 2009;280:79-83.

8. Scarmeas N, Luchsinger JA, Schupf N, et al. Physical activity, diet, and risk of Alzheimer disease. JAMA 2009;302:627-37.

9. Institute of Medicine (IOM). Cognitive aging: progress in understanding and opportunities for action. Washington, DC: The National Academies Press, 2015:109-47.

10. Wilson RS, Boyle PA, Yu L, et al. Life-span cognitive activity, neuropathologic burden, and cognitive aging. Neurology 2013;81:314-21.
11. Bosma H, van Boxtel MP, Ponds RW, et al. Engaged lifestyle and cognitive function in middle and old-aged, non-demented persons: a reciprocal association? Z Gerontol Geriatr 2002;35:575-81.

12. Hultsch DF, Hertzog C, Small BJ, et al. Use it or lose it: engaged lifestyle as a buffer of cognitive decline in aging? Psychol Aging 1999;14:245-63.

13. Wilson RS, Mendes De Leon CF, Barnes LL, et al. Participation in cognitively stimulating activities and risk of incident Alzheimer disease. JAMA 2002;287:742-8.

14. Wilson RS, Scherr PA, Schneider JA, et al. Relation of cognitive activity to risk of developing Alzheimer disease. Neurology 2007;69:1911-20.

15. Tucker AM, Stern Y. Cognitive reserve in aging. Curr Alzheimer Res 2011;8:354-60.

16. Stern Y. Cognitive reserve in ageing and Alzheimer's disease. Lancet Neurol 2012;11:1006-12.

17. Valenzuela MJ, Sachdev P. Brain reserve and dementia: a systematic review. Psychol Med 2006;36:441-54.

18. Scarmeas N, Stern Y. Cognitive reserve: implications for diagnosis and prevention of Alzheimer's disease. Curr Neurol Neurosci Rep 2004;4:374-80.

19. Diamond MC, Rosenzweig MR, Bennett EL, et al. Effects of environmental enrichment and impoverishment on rat cerebral cortex. J Neurobiol 1972;3:47-64.

20. Ngandu T, von Strauss E, Helkala EL, et al. Education and dementia: what lies behind the association? Neurology 2007;69:1442-50.

21. Marx J. Neuroscience. Preventing Alzheimer's: a lifelong commitment? Science 2005;309:864-6.

22. Fratiglioni L, Paillard-Borg S, Winblad B. An active and socially integrated lifestyle in late life might protect against dementia. Lancet Neurol 2004:3:343-53.

23. Katzman R, Terry R, DeTeresa R, et al. Clinical, pathological, and neurochemical changes in dementia: a subgroup with preserved mental status and numerous neocortical plaques. Ann Neurol 1988;23:138-44.

24. UNESCO. International Standard Classification of Education (ISCED) 2011. 2012 http://uis.unesco.org/en/glossary-term/adult-education (Accessed 11 May 2017).

25. UNESCO. Continuing education and new directions Bangkok. 1993 http://www.unesco.org/education/pdf/413_48a.pdf (Accessed 11 May 2017).

26. Manninen J, Sgier I, Fleige M. Benefits of lifelong learning in europe: main results of the bell-project. $2014 \mathrm{http}: / /$ www.bell-project.eu/cms/ wp-content/uploads/2014/06/bell-research-report.pdf (Accessed 16 May 2017).

27. OECD. Learning opportunities for adults. Participation in adult education. Paris, 1977-

28. Cedefop. Terminology of european education and training policy a selection of 100 key terms, 2008.

29. Kil M, Motschilnig R, Thöne-Geyer B. What can adult education accomplish? the benefits of adult learning - the approach, measurement and prospects. $2013 \mathrm{https}: / / \mathrm{www}$. die-bonn.de/doks/ 2013-benefits-en-01.pdf (Accessed 16 May 2017).

30. Moher D, Liberati A, Tetzlaff $\mathrm{J}$, et al. Preferred reporting items for systematic reviews and meta-analyses: the PRISMA statement. PLOS Med. 2009;89:873-80.

31. Matyas N, Auer S, Gisinger C, et al. Continuing education for the prevention of mild cognitive impairment and Alzheimer's-type dementia: a systematic review protocol. Syst Rev 2017;6:157.

32. Petersen RC. Mild cognitive impairment as a diagnostic entity. $J$ Intern Med 2004;256:183-94.

33. McGowan J, Sampson M, Salzwedel DM, et al. PRESS peer review of electronic search strategies: 2015 guideline statement. J Clin Epidemiol 2016;75:40-6.

34. Veritas Health Innovation. Covidence systematic review software. 2017 www.covidence.org.

35. Sterne JA, Hernán MA, Reeves BC, et al. ROBINS-l: a tool for assessing risk of bias in non-randomised studies of interventions. BMJ 2016;355:i4919.

36. Shea BJ, Hamel C, Wells GA, et al. AMSTAR is a reliable and valid measurement tool to assess the methodological quality of systematic reviews. J Clin Epidemiol 2009;62:1013-20.

37. Balshem $\mathrm{H}$, Helfand $\mathrm{M}$, Schünemann $\mathrm{HJ}$, et al. GRADE guidelines: 3. Rating the quality of evidence. J Clin Epidemiol 2011;64:401-6.

38. Thow ME, Summers MJ, Saunders NL, et al. Further education improves cognitive reserve and triggers improvement in selective cognitive functions in older adults: The Tasmanian Healthy Brain Project. Alzheimers Dement 2018;10:22-30.

39. Lenehan ME, Summers MJ, Saunders NL, et al. Sending your grandparents to university increases cognitive reserve: The Tasmanian Healthy Brain Project. Neuropsychology 2016;30:525-31. 
40. Di Marco LY, Marzo A, Muñoz-Ruiz M, et al. Modifiable lifestyle factors in dementia: a systematic review of longitudinal observational cohort studies. J Alzheimers Dis 2014;42:119-35.

41. Opdebeeck C, Martyr A, Clare L. Cognitive reserve and cognitive function in healthy older people: a meta-analysis. Neuropsychol Dev Cogn B Aging Neuropsychol Cogn 2016;23:40-60.

42. Sajeev G, Weuve J, Jackson JW, et al. Late-life cognitive activity and dementia: a systematic review and bias analysis. Epidemiology 2016;27:732-42.

43. Toril P, Reales JM, Ballesteros S. Video game training enhances cognition of older adults: a meta-analytic study. Psychol Aging 2014;29:706-16.

44. Yates LA, Ziser S, Spector A, et al. Cognitive leisure activities and future risk of cognitive impairment and dementia: systematic review and meta-analysis. Int Psychogeriatr 2016;28:1791-806.

45. Summers MJ, Saunders NL, Valenzuela MJ, et al. The Tasmanian Healthy Brain Project (THBP): a prospective longitudinal examination of the effect of university-level education in older adults in preventing age-related cognitive decline and reducing the risk of dementia. Int Psychogeriatr 2013;25:1145-55.

46. Ward DD, Summers MJ, Saunders NL, et al. Modeling cognitive reserve in healthy middle-aged and older adults: the Tasmanian Healthy Brain Project. Int Psychogeriatr 2015;27:579-89.
47. Akbaraly TN, Portet F, Fustinoni S, et al. Leisure activities and the risk of dementia in the elderly: results from the Three-City Study. Neurology 2009;73:854-61.

48. Verghese J, Lipton RB, Katz MJ, et al. Leisure activities and the risk of dementia in the elderly. N Engl J Med 2003;348:2508-16.

49. Pascual-Leone A, Amedi A, Fregni $F$, et al. The plastic human brain cortex. Annu Rev Neurosci 2005;28:377-401.

50. Knaepen K, Goekint M, Heyman EM, et al. Neuroplasticity - exerciseinduced response of peripheral brain-derived neurotrophic factor: a systematic review of experimental studies in human subjects. Sports Med 2010;40:765-801.

51. Vance DE, Roberson AJ, McGuinness TM, et al. How neuroplasticity and cognitive reserve protect cognitive functioning. J Psychosoc Nurs Ment Health Serv 2010;48:23-30.

52. Norton S, Matthews FE, Barnes DE, et al. Potential for primary prevention of Alzheimer's disease: an analysis of population-based data. Lancet Neurol 2014;13:788-94.

53. Ngandu T, Lehtisalo J, Solomon A, et al. A 2 year multidomain intervention of diet, exercise, cognitive training, and vascular risk monitoring versus control to prevent cognitive decline in at-risk elderly people (FINGER): a randomised controlled trial. Lancet 2015;385:2255-63. 